Monatsschr Kinderheilkd 2020 • 168:842-844 https://doi.org/10.1007/s00112-019-00813-x Online publiziert: 28. Februar 2020

(c) Der/die Autor(en) 2019

\section{Redaktion}

A. Borkhardt, Düsseldorf

S. Wirth, Wuppertal

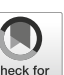

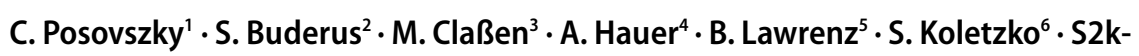
Leitliniengruppe akute infektiöse Gastroenteritis im Säuglings-, Kindes- und Jugendalter

' Klinik für Kinder- und Jugendmedizin, Universitätsklinikum Ulm, Ulm, Deutschland

${ }^{2}$ Pädiatrie, St. Marienhospital Bonn, Bonn, Deutschland

${ }^{3}$ Gesundheit Nord gGmbH Klinikverbund Bremen, Bremen, Deutschland

${ }^{4}$ Universitätsklinik für Kinder- und Jugendheilkunde, Medizinische Universität Graz, Graz, Österreich

${ }^{5}$ Praxis für Kinder- und Jugendmedizin, Arnsberg, Deutschland

${ }^{6}$ Dr. von Haunersches Kinderspital, Ludwig-Maximilians-Universität, München, Deutschland

\title{
Handlungsempfehlung nach der „S2k-Leitlinie akute infektiöse Gastroenteritis im Säuglings-, Kindes- und Jugendalter"
}

beiden Lebensjahren sowie Kinder und Jugendliche nach ausgedehnten Darmresektionen oder mit Immundefizienz, Diabetes mellitus, Stoffwechseldefekten oder Malnutrition. Zeichen einer vitalen Gefährdung sind: eingeschränktes Bewusstsein; kaltschweißige Haut; extrem eingesunkene Augen und ggf. Fontanelle; trockene Schleimhäute; fehlende Tränen; schlaffer Muskeltonus; $>6$ bzw.10\% Verlust des Körpergewichts; verlängerte kapilläre Füllungszeit (>3s); Tachypnoe; Acidose-Atmung; Tachykardie; Anurie.
Als Standardtherapie bei leicht- bis mittelgradiger Dehydratation soll rasch bereits ambulant eine orale Rehydrierung unter ärztlicher Supervision mithilfe von glucose- oder polymerbasierten Elektrolytlösungen erfolgen. Eine nasogastrale Sonde sollte zur enteralen Rehydrierung gelegt werden, wenn die orale Rehydrierung durch häufiges Trinken oder Löffeln kleiner Volumina nicht gelingt (• Abb. 1). Eine i.v.-Rehydrierung soll bei Scheitern einer oralen bzw. nasogastralen Rehydrierung, bei Schockzustand, bei schwerenden Faktoren (• Abb. 1; [1]). Die Leitsymptome einer AGE sind plötzliche Minderung der Stuhlkonsistenz und Steigerung der Stuhlfrequenz sowie evtl. Erbrechen oder Fieber. Eine Arztvorstellung soll aufgrund des erhöhten Komplikationsrisikos bei allen Säuglingen, persistierendem Erbrechen, Trinkverweigerung $>4 \mathrm{~h}$, Vorliegen einer schweren Grunderkrankung, Lethargie, hohem Fieber, starken Bauchschmerzen, großvolumigen oder blutigem Durchfall erfolgen. Die Erfassung des Schweregrads der Dehydratation bildet die Basis der Behandlung (•Tab. 1 und 2). Besonders gefährdet sind Kinder in den ersten

Die Mitglieder der S2k Leitliniengruppe „Akute infektiöse Gastroenteritis im Säuglings-, Kindesund Jugendalter" werden am Beitragsende gelistet.

\begin{tabular}{|c|c|c|}
\hline Parameter & Klinische Befunde & Score \\
\hline \multirow{3}{*}{$\begin{array}{l}\text { Allgemeines } \\
\text { Erschei- } \\
\text { nungsbild }\end{array}$} & Normal & 0 \\
\hline & Durstig, unruhig oder lethargisch, aber irritabel, wenn berührt & 1 \\
\hline & Taumelig, kaltschweißig, komatös & 2 \\
\hline \multirow[t]{3}{*}{ Augen } & Normal & 0 \\
\hline & Leicht eingesunken & 1 \\
\hline & Extrem eingesunken & 2 \\
\hline \multirow{3}{*}{$\begin{array}{l}\text { Schleimhäute, } \\
\text { Zunge }\end{array}$} & Feucht & 0 \\
\hline & Klebrig & 1 \\
\hline & Trocken & 2 \\
\hline \multirow[t]{3}{*}{ Tränen } & Vorhanden & 0 \\
\hline & Wenig Tränen & 1 \\
\hline & Keine Tränen & 2 \\
\hline
\end{tabular}




\section{Leitsymptome für der akuten infektiösen Gastroenteritis}

Red Flags für Arztvorstellung:

Säugling, Trinkverweigerung $>4 \mathrm{~h}$, persistierendes Erbrechen, schwere Malnutrition, relevante Grunderkrankung (z.B. Darmresektion, Immundefizienz, Stoffwechselerkrankung, Typ-1-Diabetes), Lethargie, hohes Fieber, starke Bauchschmerzen, großvolumiger, blutiger oder anhaltender Durchfall

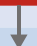

Klinische Evaluation u.a. Gewichtsverlust, KFZ, KDS

올

\section{Ke}

Keine Dehydratation

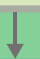

Prävention der Dehydratation

- Stillen bzw. altersgemäße Nahrung

- Ausreichend Flüssigkeitszufuhr

-ggf. ORL
Grad der Dehydration

(Tab. 2)
Häusliche Versorgung

- Information zu

Krankheitsverlauf,

Flüssigkeitszufuhr

- Rezept für ORL

- Evtl. Verlaufskontrolle

- Altersgemäße Ernährung

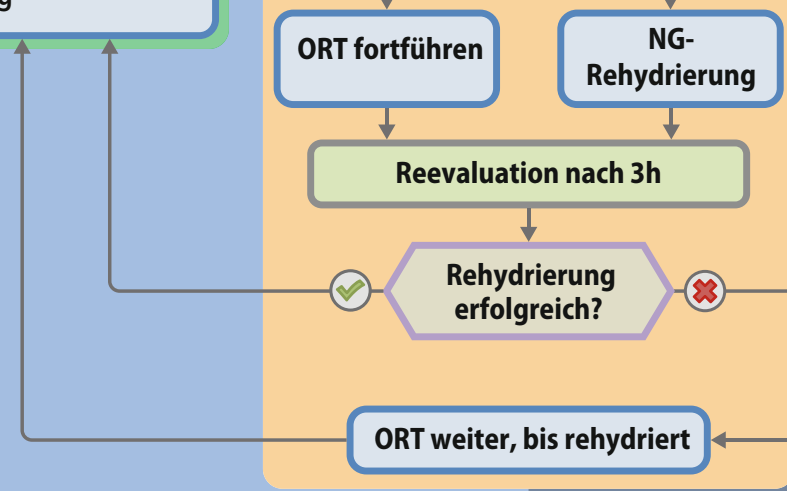

Ambulant

\section{Faktoren für stationäre}

Versorgungsbedürftigkeit:

Säuglinge < 3500 gKG od. jünger als 2 Monate, nicht gesicherte ambulante Umsetzung, gescheiterte orale Rehydrierung; Schock / schwere Acidose; Hypo-/Hypernatriämie $\left(\mathrm{Na}^{+}<130 \mathrm{bzw} .>150 \mathrm{mmol} / \mathrm{l}\right)$; neurologische

Symptome (Lethargie/Koma); schwere (chronische) Grunderkrankung, Malnutrition oder Gedeihstörung; V.a. lleus / intestinale Transportstörung; anhaltend blutige Diarrhö; V.a. andere Ursache der Symptomatik

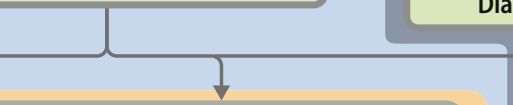


Abb. 1 Therapeutisches Vorgehen bei akuter infektiöser Gastroenteritis entsprechend der klinischen Dehydratation. Cave: Für Säuglinge und Kinder gelten unterschiedliche Grenzwerte des prozentualen Gewichtsverlusts in Bezug auf den Grad der Dehydratation (•Tab. 2). BE Blutentnahme; $B B$ Blutbild; Cl Chlorid; Gluc Glucose; HSt Harnstoff; KKalium; KDS klinischer Dehydratations-Score (- Tab. 1); KFZ kapilläre Füllungszeit; Krea Kreatinin; Na Natrium; NG nasogastral; ORL orale Rehydrierungslösung; ORT orale Rehydrierungstherapie; SBS Säure-Base-Status. (Nach Gesellschaft für Pädiatrische Gastroenterologie und Ernährung e.V [1]; Grafik: O. Hippmann)

Tab. 2 Grad der Dehydratation

\begin{tabular}{|c|c|c|c|c|}
\hline \multirow[t]{2}{*}{ Parameter } & \multicolumn{4}{|c|}{ Dehydratation } \\
\hline & Keine & Leicht & Mittelgradig & Schwer \\
\hline \multicolumn{5}{|c|}{ Gewichtsverlust (\%) } \\
\hline - Säugling & 0 & $\leq 5$ & $6-10$ & $>10$ \\
\hline - Kind & 0 & $\leq 3$ & $4-6$ & $>6$ \\
\hline KFZ (s) & $<2$ & 2 & $3-4$ & $>4$ \\
\hline KDS-Summe & 0 & $1-4$ & & $5-8$ \\
\hline
\end{tabular}

rer Dehydratation oder bei Symptomen eines Ileus erfolgen.

Die vorliegende Handlungsempfehlung beruht auf der „S2k-Leitlinie akute infektiöse Gastroenteritis im Säuglings-, Kindes- und Jugendalter" der Arbeitsgemeinschaft der Wissenschaftlichen Medizinischen Fachgesellschaften e.V. (AWMF, [1, 2]). Die Handlungsempfehlung ersetzt nicht die Lektüre der Leitlinie.

\section{Korrespondenzadresse}

\section{PD Dr. C. Posovszky}

Klinik für Kinder- und Jugendmedizin, Universitätsklinikum Ulm

Eythstr. 24, 89075 Ulm, Deutschland

Carsten.Posovszky@uniklinik-ulm.de

Mitglieder der S2k-Leitliniengruppe akute infektiöse Gastroenteritis im Säuglings-, Kindes- und Jugendalter. C. Posovszky; V. Backendorf; S. Buderus; M. Claßen; H.-J. Epple; B. Gruber; A.C. Hauer; J. Hübner; K.M. Keller; S. Koletzko; B. Lawrenz; A. Schmidt-Choudhury; A. Stallmach; U. von Both

\section{Einhaltung ethischer Richtlinien}

Interessenkonflikt. C. Posovszky, S. Buderus, M. Claßen, A. Hauer, B. Lawrenz und S. Koletzko erhielten Honorare für Vortrags- und Schulungstätigkeiten oder bezahlte Autoren-oder Co-Autorenschaften, als Berater oder Gutachter sowie finanzielle Zuwendungen für Forschungsvorhaben eines Unternehmens der Gesundheitswirtschaft oder kommerziell orientierten Auftragsinstituts. Eine ausführliche Darstellung und Offenlegung der Interessenkonflikte der einzelnen
Autoren findet sich im Leitlinienreport. Interessenkonflikte hinsichtlich oraler Rehydrationslösungen und Probiotika wurden durch Stimmenthaltung berücksichtigt. Gelegentliche Honorare und Vortragstätigkeiten wurden als geringfügige Interessenkonflikte gewertet.

Für diesen Beitrag wurden von den Autoren keine Studien an Menschen oder Tieren durchgeführt. Für die aufgeführten Studien gelten die jeweils dort angegebenen ethischen Richtlinien.

Open Access. Dieser Artikel wird unter der Creative Commons Namensnennung 4.0 International Lizenz (http://creativecommons.org/licenses/by/4.0/deed. de) veröffentlicht, welche die Nutzung, Vervielfältigung, Bearbeitung, Verbreitung und Wiedergabe in jeglichem Medium und Format erlaubt, sofern Sie den/die ursprünglichen Autor(en) und die Quelle ordnungsgemäß nennen, einen Linkzur Creative Commons Lizenz beifügen und angeben, ob Änderungen vorgenommen wurden.

\section{Literatur}

1. Gesellschaft für Pädiatrische Gastroenterologie und Ernährung e.V. (2019) S2k Leitlinie Akute infektiöse Gastroenteritis im Saeuglings, Kindesund Jugendalter AWMF

2. Posovszky C, Backendorf V, Buderus S, Classen M, Epple H-J, Gruber B, Hauer AC, Hubner J, Keller KM, Koletzko S, Lawrenz B, Schmidt-Choudhury A, Stallmach A, von Both U (2019) S2k-Leitlinie „Akute infektiöse Gastroenteritis im Säuglings-, Kindesund Jugendalter" - AWMF Registernummer 068003.Z Gastroenterol 57(9):1077-1118. https://doi. org/10.1055/a-0981-6906

3. Friedman JN, Goldman RD, Srivastava R, Parkin PC (2004) Development of a clinical dehydration scale for use in children between 1 and 36 months of age. JPediatr 145:201-207 